\title{
First experience of seismodeformation monitoring of Baikal rift zone (by the example of South-Baikal earthquake of 27 August 2008)
}

\author{
G. V. Vstovsky ${ }^{1}$ and S. A. Bornyakov ${ }^{2}$ \\ ${ }^{1}$ All Russia Research and Design Institute of Atomic Machinery (Joint Stock Company "VNIIAM"), Moscow, Russia \\ ${ }^{2}$ Institute of Earth's Crust, Siberian Department of Russian Academy of Sciences, Irkutsk, Russia
}

Received: 11 November 2009 - Accepted: 19 March 2010 - Published: 7 April 2010

\begin{abstract}
A novel method of data processing - a structural functions curvature analysis method - was applied to the time series of seismodeformation monitoring of Baikal rift zone from April to November 2008, revealing the unique features of monitoring variable behaviour that can be considered as a revelation of precursors to the intensive South-Biakal earthquake ( $M=6.3$, at 09:31 on 27 August 2008). The idea of a new approach leans upon basic ideas of modern physics of self-organized criticality and open non-equilibrium systems in general.
\end{abstract}

\section{Introduction}

The advances in experimental seismology during the $70 \mathrm{~s}$ and 80 s of the last century had stimulated the development of instrumental works in geodynamic testing areas. Taking into account the deformation nature of earthquakes and the preceding geophysical phenomena, great attention had been paid to deformation monitoring. Its technical base had been improved step-by-step and this was a way from simple manually controlled mechanical constructions to complex tensometric laser and other automatic installations enabling us to obtain long-term deformation variable time series with required discretization.

The development of a new synergetic concept of open systems evolution (Haken, 1983; Kondepudi and Prigogine, 1999) and its application to geological and seismological investigations (Letnikov, 1992; Gol'din, 2005; Keilis-Borok, 1990; Bak, 1989; Sornette et al., 1989, 1995; Saleur et al., 1996; Heimpel, 1997; Bowman et al., 1998; Hainzl et al., 2000; Grasso et al., 1998; Zoller et al., 2002) made it possible

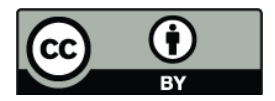

Correspondence to: G. V. Vstovsky (vstovsky@yandex.ru) to develop an advanced methodology. It is aimed at disclosing and analysing the hierarchical structure of geophysical systems. One of many approaches of such a methodology is the analysis of correlation times (CT) of local processes investigated by stochastic time series of geophysical variables. It is implied (Vstovsky, 2008) that, as the processes take place on/in the distributed self-organized system, the changes in the state of such a system must influence the course of the local processes. For instance, during the accumulation of deformation in the Earth's crust under external and internal factors, and as a consequence, the rearrangement of the crust space structure, CT of local deformation processes can change. The registration and adequate interpretation of such changes can be an effective tool for the monitoring of the states of both the separate regions and the lithosphere as a whole to reveal and forecast the transition and critical states in the lithosphere evolution process.

A structural functions curvature analysis method (SFCAM) provides an effective tool for time series analysis (Vstovsky, 2008, 2006), enabling us to reveal the CT hierarchy on the basis of a simple quick operating mathematical apparatus.

In this work, the first experience of SFCAM application to the processing of seismo-deformation data on seismically active Baikal rift zone (BRZ) is described.

\section{Monitoring location and technique}

Monitoring equipment was placed in an adit located in the region of "Talai" seismostation of the Biakal branch of Geophysical Service of the Siberian Department of Russian Academy of Sciences. It was a probe adit made of marble in the late 1950s but later, being unclaimed, it was equipped for scientific goals and now it is used for monitoring of different types. The adit is characterised by constant temperature and humidity.

Published by Copernicus Publications on behalf of the European Geosciences Union. 
(a)

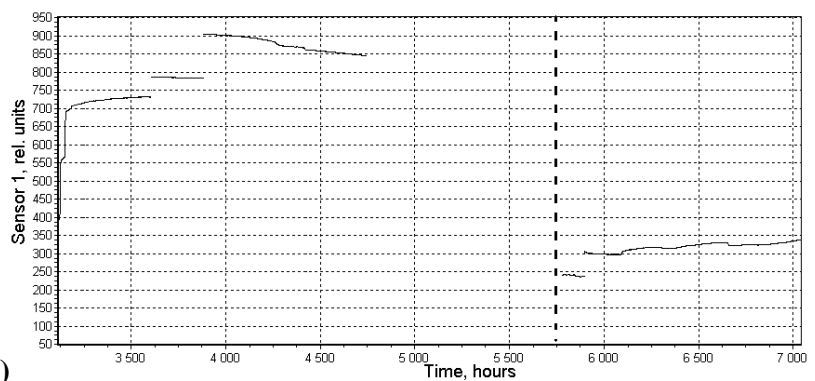

(b)
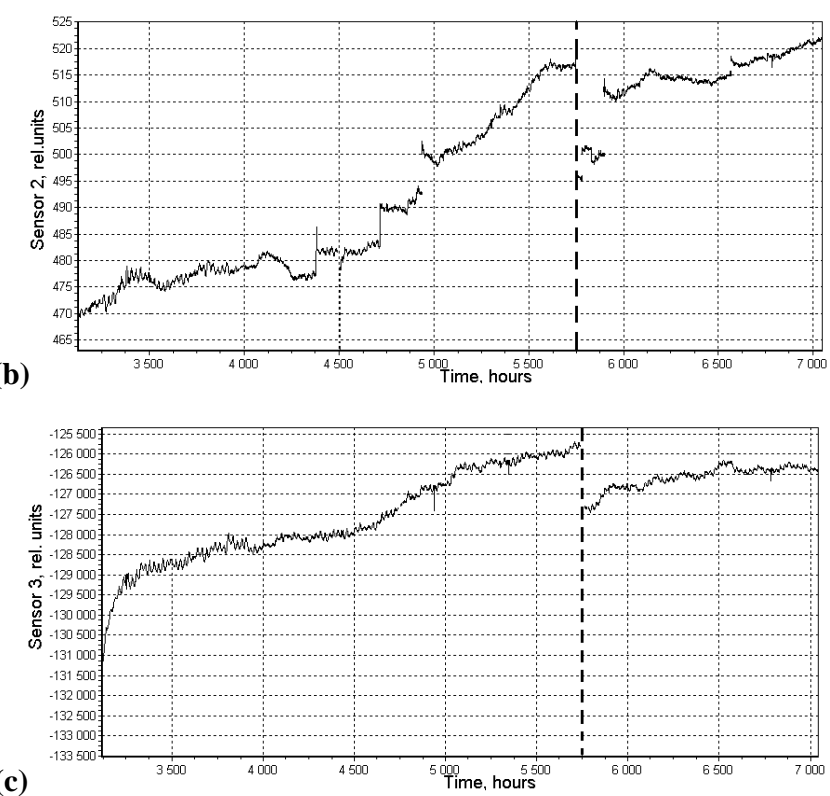

Fig. 1. Data of sensors 1, 2, 3 (a, b, c, respectively). Hour samplings are shown. Earthquake beginning is marked by dotted line.

Monitoring was carried out since April to November 2008. An intensive South-Biakal earthquake $(M=6.3$, at 09:31 on 27 August 2008) took place during monitoring time. During this period, the automatic instrumental measurements of massive rock deformations were carried out in longitudinal, normal (with spreadings $150^{\circ}$ and $60^{\circ}$ ) and vertical directions, sensors $1,2,3$, respectively. The original bar sensors were used with a base $1.8 \mathrm{~m}$ and the ends fixed at the adit walls. Their active sensitive elements were beam type strain sensors by the "Scame" company. Sensors were placed at such marble regions that had no cracks. 30 s sensor sampling, digital data accumulation and storing was conducted by the "Sdvig" recorder (Ruzhich, 2004). The recorded signals are shown in Fig. 1 as hour samplings. The time coordinate is given in hours from the beginning of the year. The earthquake beginning $(5745.5 \mathrm{~h})$ is marked by dotted line.

There were gapes in the data, due to technical reasons. The most significant gaps were in the data of (longitudinal) sensor 1 during the period just before the earthquake, so the results obtained by the data of sensors 2 and 3 were used to obtain the results discussed below.

\section{Data processing method and results}

The data were processed by the combination of SFCAM and sliding window method (SWM). The latter enables us to present dynamical variable series as a sequence of values of one or another parameter calculated for each position of a data window of a given length. The window time coordinate is a position of its forward boundary. For each window position a structural function (SF) of order $p$ was calculated by formula

$\Phi^{p}(\Delta)=\frac{1}{M} \sum_{k=1}^{M}\left|h\left(t_{k}\right)-h\left(t_{k}+\Delta\right)\right|^{p}$,

where $h(t)$ - signal value in the window of $N$ samples ("points"), the signal being given discretely in points $t_{k}=$ $k \Delta t$ ( $\Delta t$ - discretization step) along coordinate $t, M=N-$ $\Delta / \Delta t, \Delta-$ lag (argument) of SF equal to $\Delta t, 2 \Delta t, 3 \Delta t, \ldots$, $\left(N_{\Phi}\right) \Delta t, N_{\Phi}=(0.5-0.8) N, p-$ SF order. $p=2$ is used in the following and this index will be omitted. SF represents an effective tool in theoretical and experimental investigations of chaotic systems and processes, for example, in turbulence investigations (Frisch, 1995).

Figure 2 explains SFCAM. It shows examples of signal window, its SF (in double logarithmic axes) and the corresponding negative second derivative of smoothed SF. Extremuma positions of the latter give the evaluations of CTs.

The physical sense of SF is described here. The SF growth for small lags $\Delta$ means that there are correlation links, interrelations, in the signal. The cessation of SF growth, saturation, for large lags means failure (lost) of the interrelations. The conventional boundary of these scale regions (determined by one or another rule or algorithm) is called a correlation time (CT). In the simplest cases, when SF has only one "footstep", there is only one type of interrelation characterized by CT according to the "footstep position" which can be determined by the position of extremum negative derivative of smoothed SF (under the condition of positive first derivative) that was first proposed in (Vstovsky, 2006). That paper demonstrated the effectiveness of such an approach in comparison with many parameter fitness (approximation) of SF. When SF has several "footsteps", corresponding to several interrelations with different CTs, the evaluation of SF's negative second derivatives extremuma positions enables us to evaluate such CTs easily. Therefore, we can evaluate hierarchy of CTs. The case of SF with three "footstep" is shown in Fig. $2 \mathrm{~b}$ and c. CTs are denoted by $T_{1}, T_{2}, T_{3}, \ldots$ starting with the smallest.

Since real data are measured with finite accuracy, fluctuations and with other reasons for imperfect, not smooth SF, we have to calculate CTs by a special multi-step algorithm including operations of reinterpolation of double logarithmic SF, smoothing it and taking its second derivative. The algorithm enables us to take into account the presence of data gaps (marked with special value) that are unique advances of the procedures used. 
It should be stressed that the use of SF enables us, in this case, to reveal the presence of long term correlations with CT up to $1700 \mathrm{~h}$ (about 70 days) that corresponds to SF growth for lags larger than $1000 \mathrm{~h}$. Such correlations witnessed concerning the strong non-stationarity of the processes under investigation and local instability of the lithosphere during the period before the disaster earthquake. The ability of the method to reveal such qualitative features of the system under study, shows in favour of the proposed approach.

The data were processed by a specially developed program called SHIFT (Scale Hierarchy Information Fertile Treatment), which enables the calculation of SFs, and their second derivatives, with the determination of CTs for any position of a window of given length and to calculate the dependence of the parameters on time. The used window size was 1000 samples $(1000 \mathrm{~h}=41.67$ days $)$. The window shift step was 20 samples. The maximum SF lag used here was $800 \mathrm{~h}$ ( $80 \%$ of window size).

Besides using SFCAM, the non-stationarity criteria were calculated to confirm the moments of significant changes in the system's behaviour, registered by rearrangements of SFs and, consequently, by sharp changes of CTs. Let $\Phi^{k}\left(\Delta_{i}\right)$ $\left(\Delta_{i}=i^{*} \Delta t\right.$ ) be $i$-th SF value calculated for $k$-th position of the sliding window. One non-stationarity criterion set proposed by Prof. Timashev (Descherevsky et al., 2003) (integral relative criterion - IRC) reads

$$
\begin{aligned}
\operatorname{IRC}^{k}= & \frac{\sum_{i} \Phi^{k}\left(\Delta_{i}\right)-\sum_{i} \Phi^{k-1}\left(\Delta_{i}\right)}{\sum_{i} \Phi^{k-1}\left(\Delta_{i}\right)}, \\
\operatorname{IRC}_{1}^{k}= & 2 \frac{\sum_{i} \Phi^{k}\left(\Delta_{i}\right)-\sum_{i} \Phi^{k-1}\left(\Delta_{i}\right)}{\sum_{i} \Phi^{k}\left(\Delta_{i}\right)+\sum_{i} \Phi^{k-1}\left(\Delta_{i}\right)} .
\end{aligned}
$$

Another criterion set used in this work - relative integral criterion (RIC) - reads

$$
\begin{aligned}
\operatorname{IRC}^{k} & =\sum_{i} \frac{\Phi^{k}\left(\Delta_{i}\right)-\Phi^{k-1}\left(\Delta_{i}\right)}{\Phi^{k-1}\left(\Delta_{i}\right)}, \\
\operatorname{IRC}_{1}^{k} & =2 \sum_{i} \frac{\Phi^{k}\left(\Delta_{i}\right)-\Phi^{k-1}\left(\Delta_{i}\right)}{\Phi^{k}\left(\Delta_{i}\right)+\Phi^{k-1}\left(\Delta_{i}\right)} .
\end{aligned}
$$

Values (2) must be approximately zero for stationary processes. Sharp changes of values (2), when window "slides" along the series, characterize the extent of non-stationarity of the processes under study. Criteria (2) can also be generalized for the use of other window functions such as power spectra, correlation functions, etc. instead of SF. But the experience of their use showed effectiveness of criteria (2). (a)

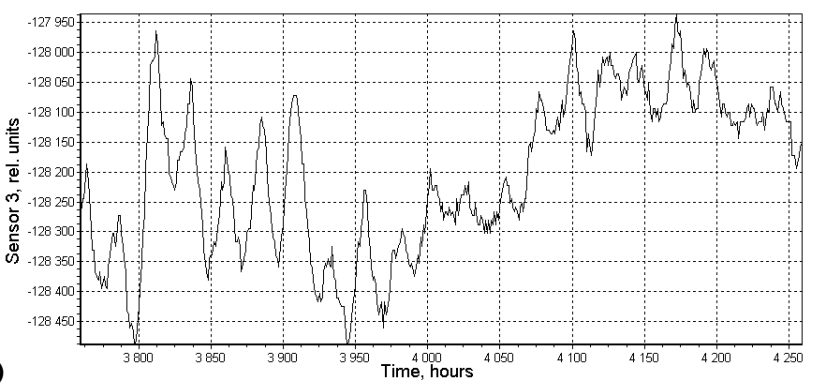

(b)

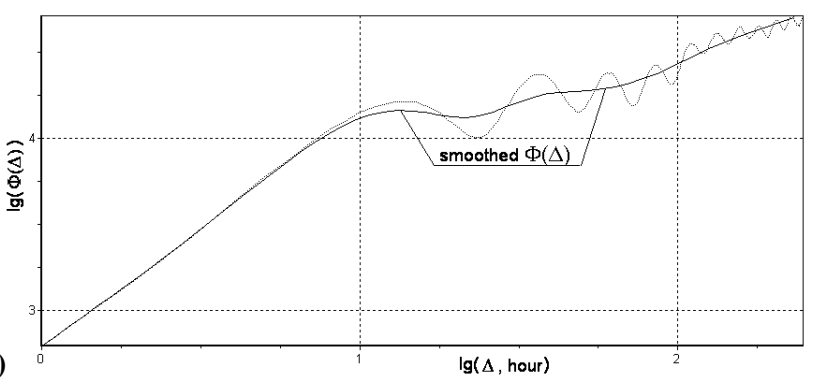

(c)

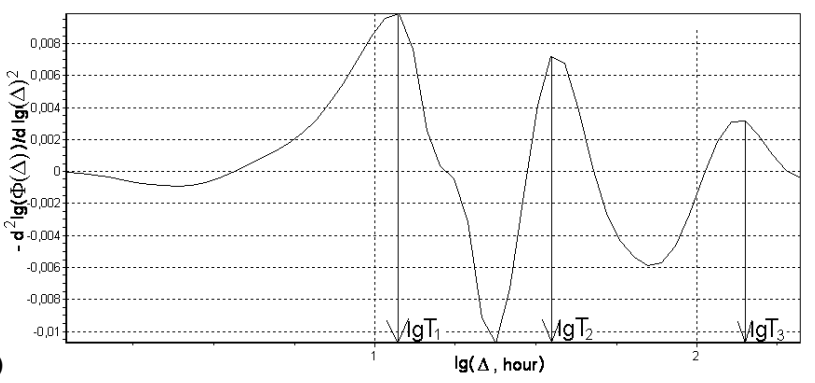

Fig. 2. SFCAM explanation. Window of sensor 3 signal (a), its double logarithmic SF and smoothed double logarithmic SF (b) and negative second derivative ("curvature") of smoothed double logarithmic SF (c) with indication of extremuma positions which give CTs evaluations.

\section{Results and discussion}

Figure 3 shows the results of CTs $T_{1}, T_{2}, T_{3}$ calculation by data of sensors 2 and 3 (the normal and vertical directions, respectively). As is seen, the sharp changes of CTs of local lithosphere deformation processes are observed approximately at $1200 \mathrm{~h}$ (by sensor 2 data) and at $800-1000 \mathrm{~h}$ (by sensor 3 data) before the earthquake. Such a behaviour witnesses the destruction of time correlations of the endogenic processes in the Earth crust that are due to the instability of the lithosphere space structure that results in significant rearrangement of stress fields in the lithosphere and the preparation of possible disaster rearrangement of the lithosphere structure, i.e. it results in an earthquake. Thus, we can say, in this case, about revelation of midterm (about $1100 \mathrm{~h}, 46$ days) precursor of the disaster earthquake.

More exactly, a state with CTs $T_{1}=10 \mathrm{~h}, T_{2}=30-40 \mathrm{~h}$, $T_{3}=150 \mathrm{~h}$ for both sensors changes by a state with $T_{1}=2-4 \mathrm{~h}$ 
(a)

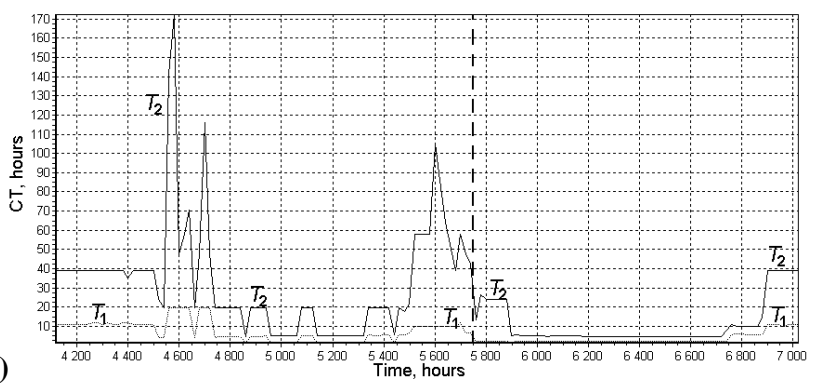

(b)

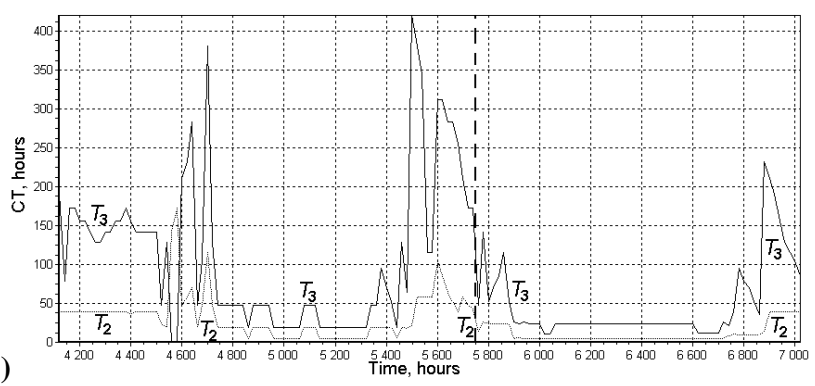

(c)
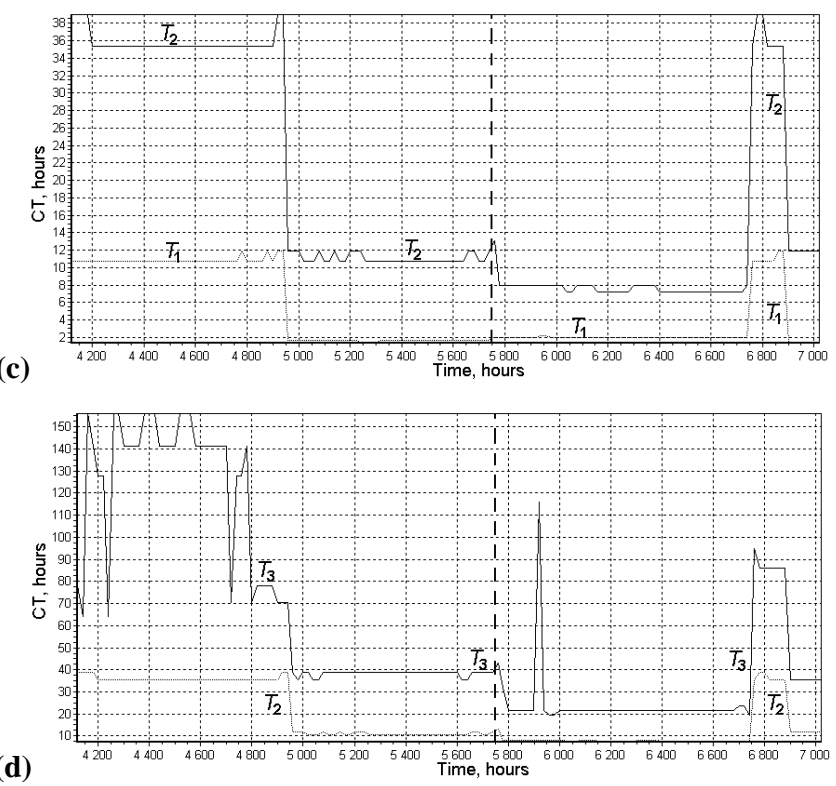

Fig. 3. Results of calculation CTs $T_{1}, T_{2}(\mathbf{a}, \mathbf{c})$ and $T_{2}, T_{3}(\mathbf{b}, \mathbf{d})$ by sensors $2(\mathrm{a}, \mathrm{b})$ and 3 (c, d) data. Earthquake is marked by dotted line.

(both sensors), instable $T_{2}=5-20 \mathrm{~h}$ (sensor 2) and $T_{2}=10 \mathrm{~h}$ (sensor 3), instable $T_{3}=20-50 \mathrm{~h}$ (sensor 2 ) and $T_{3}=40 \mathrm{~h}$ (sensor 3 ) at $4800-5000 \mathrm{~h}$, i.e. at $1000-800 \mathrm{~h}$ before the earthquake. CTs $T_{2}, T_{3}$ of normal deformations (sensor 2) have sharp oscillations in a wide range and reach great values $T_{2}=170 \mathrm{~h}, T_{3}=365 \mathrm{~h}$ during such a transition. They also have another wide range of oscillations at $300 \mathrm{~h}$ before the earthquake. A state with $T_{1}=1-2 \mathrm{~h}, T_{2}=6-8 \mathrm{~h}, T_{3}=20-22 \mathrm{~h}$ for both sensors takes place in $200 \mathrm{~h}$ after the earthquake. So, we can describe the registration of the system's "switching" into another state. The decrease in all the CTs by 7-8 times (a)
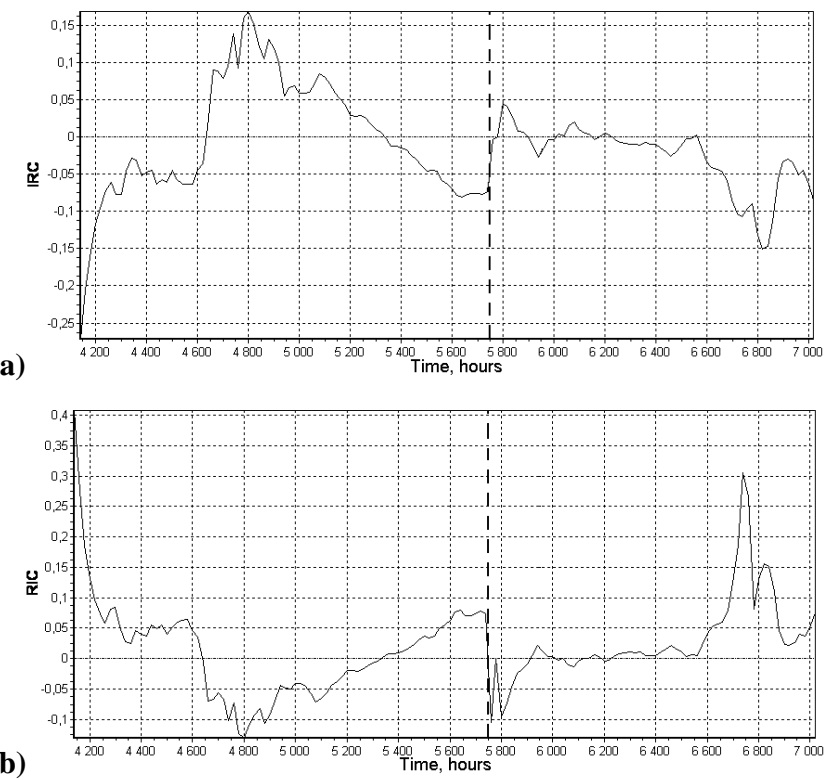

Fig. 4. The integral relative (IRC, a) and relative integral (RIC, b) non-stationarity criteria for sensor 3 data. $\mathrm{IRC}_{1}$ and $\mathrm{RIC}_{1}$ criteria are almost the same in this case (but this is not true in general).

describes a strong rearrangement of the space structure of the system under study. At 7000 h, CTs become almost the same as at the beginning of observations after the transition through another instability for 6750-6900 h. It should be noted that CT's changes just after a strong earthquake are hard to interpret as a precursor of the following events while the system is in an unstable state that can last for a year and longer.

The revealed moments of the system instability are confirmed by the results of the calculations of non-stationarity criteria (2). Figure 4 shows the criteria for vertical deformations (sensor 3 ) data. The registered moments of instability are almost the same - about $4800 \mathrm{~h}$, very earthquake $(5746 \mathrm{~h})$ and about $6800 \mathrm{~h}$.

BRZ represents an extended rift zone formed at the boundary of the Euroasia and Amur plates. High velocities of their relative movement (up to $10 \mathrm{~mm} /$ year and faster) provides the level of stresses in BRZ lithosphere, enough for the development of modern process of rift formation accompanied by high seismic activity.

The authors propose the following interpretation of the results obtained, using an idea of self-organized criticality (Bak et al., 1988). Any large fault zone is an open non-equilibrium dynamical system and its evolution represents a synergetic process of slow preparation and following fast relaxation of the structural transformations of the internal systems (structures) of failures and discontinuity flaws (Bornyakov et al., 2004, 2008). This process flows step-by-step at all the structural levels under the conditions of progressive deformation. 
As a rule, two, as a minimum, structural rearrangements forego the act of appearance of a single backbone break owing to the merging of multiple advance smaller failures (Seminsky, 2003). A universal feature, of the systems of advance failures, is their ability to self-organization in the critical state before structural rearrangements, resulting in rift dissipative structures (RDS) (Bornyakov et al., 2004, 2008). The characteristic features of their deformation processes are the coherence and autowave regime, i.e. the properties peculiar to lithosphere in metastable preseismogenic state, the properties reflecting the presence of cooperative phenomena (Gol'din, 2005; Keilis-Borok, 1990; Sobolev et al., 2003).

Following to general speculations above, it can be supposed that several months before the South-Baikal earthquake the lithosphere in South Baikal region proceeded into a metastable state with formed RDS. The process of segmentation of the discontinuity flaw system, developing in RDS, is rather transient, runs in stages in reverse direction from the larger scales to the smaller ones (Bornyakov, 2008). Following the results obtained, approximately $1-1.5$ months before South-Baikal earthquake, a process of self-organization and rearrangement of active segments network took place (approximately 49 to 27 days before the earthquake). According to Sobolev et al. (2003); Sobolev (2008), the region of lithosphere metastable state propagation can exceed the "nidus" region by an order and more, i.e. it can be hundreds of kilometers.

\section{Conclusions}

The results, obtained in this work, describe both the possibility of applying the proposed approach to disclose strong earthquake precursors and the rightfulness of seismic events analysis on the basis of general methodology of open systems self-organization theory. The combination of permanent monitoring using the described tools and registration system with data processing on the basis of SFCAM and SWM can be considered for the perspective elaboration of an effective tool for preventive notification on the possible strong, seismic disaster events that can reduce the negative after-effects on responsible industrial and civil objects. The latter circumstance is of great importance now when there is an increase in seismic activity of the Earth's crust during the last 10-15 years that compels us to reconsider the maps of seismic zoning.

Acknowledgements. The basic ideas of SFCAM were born after several years of dealing with a so called Flicker Noise Spectroscopy - a method of series processing which had been developed by Prof. S. F. Timashev. Authors thank Department of the Earth Sciences of RAS and Russian Fund of Basic Research for financial support of the field studies (Projects ONZ-7.7; RFBR 10-05-00678-a).

Edited by: M. E. Contadakis

Reviewed by: S. Sherman and another anonymous referee

\section{References}

Bak, P. and Tang, C.: Earthquake as a self-organized critical phenomenon, J. Stat. Phys., 54, 1441-1458, 1989.

Bak, P., Tang, C., and Winselfield, K.: Self-organized criticality, Phys. Rev. A, 38, 364-375, 1988.

Bornyakov, S. A., Cheremnykh, A. V., and Truskov, V. A.: Dissipative structures of rift zones and criteria of their diagnostics (by the results of physical modeling), Russ. Geol. Geophys.+, 49(N2), 179-187, 2008.

Bornyakov, S. A., Gladkov, A. S., Adamovich, A. N., Matrosov, V. A., and Klepikov, V. A.: Nonlinear dynamics of rift formation by the results of physical modeling, Geotechtonics, N5, 85-95, 2004.

Bowman, D., Ouillon, G., Sammis, C., Sornette, A., and Sornette, D.: An observational test of the critical earhquke concept, J. Geophys. Res., 103, 24359-24372, 1998.

Descherevsky, A. V., Lukk, A. A., Sidorin, A. Y., Vstovsky, G. V., and Timashev, S. F.: Flicker-noise spectroscopy in earthquake prediction research, Nat. Hazards Earth Syst. Sci., 3, 159-164, 2003

Frisch, U.: Turbulence. The Legacy of A. N. Kolmogorov, Cambtidge Univ. Press, 1995.

Gol'din, S. V.: Macro - and Mezostructures of focal earthquake region, Physical mezomechanics, Novosibirsk, 8(N1), 5-14, 2005.

Grasso, J.-R. and Sornette, D.: Testing self-organized criticality by induced seismisity, J. Geophys. Res., 103, 29965-29987, 1998.

Hainzl, S., Zoller, G., and Kurths, J.: Seismic quinscence as an indicator for large earthquakes in a system of self-organized criticality, Geophys. Res. Lett., 27, 597-600, 2000.

Haken, H.: Synergetics, an Introduction: Nonequilibrium Phase Transitions and Self-Organization in Physics, Chemistry, and Biology, 3rd rev. enl. edn., Springer-Verlag, New York, 1983.

Heimpel, M.: Critical behavior and the evolution of fault strength during earthquake cycles, Nature, 338, 865-868, 1997.

Keilis-Borok, V. I.: The lithosphere as non-linear system with implications for earthquake prediction, Rev. Geophys., 28, 19-34, 1990.

Kondepudi, D. and Prigogine, I.: Modern thermodynamics: from heat engine to dissipative structure, 1999.

Letnikov, F. A.: Synergetics of Geological Systems, Novosibirsk, Nauka, 1992 (in Russian).

Ruzhich, V. V.: High-frequency measuring complex "Sdvig". Scientific and industrial potential of Siberia: Investment projects, new technologies and elaborations, International catalogue, Novosibirsk, 90-91, 2004.

Saleur, H., Sammis, C., and Sornette, D.: Discrete scale invariance, complex fractal dimensions, and log-periodic fluctuations in seismisity, J. Geophys. Res., 101, 17661-17667, 1996.

Seminsky, K. Zh.: Internal structure of continental rift zones: tectonophysical aspect, Novosibirsk, Siberian Dept. of RAS, 2003.

Sobolev, G. A., Lyubushin, A. A., and Zakrizhevskaya, N. A.: Asymmetric pulses, periodicity and synchronization of lowfrequency micriseisms, J. Volcanol. Seismol.+, N2, 135-152, 2008.

Sobolev, G. A. and Ponomarev, A. V.: Earthquake physics and precursors, Moscow, Nauka, 2003.

Sornette, A. and Sornette, D.: Self-organized criticality and earthquakes, EPL-Europhys. Lett., 9, 197-202, 1989. 
Sornette, D. and Sammis, C.: Complex critical exponents from renormalization theory group of earthquakes: Implication for earthquake prediction, J. Phys. I., 5, 607-619, 1995.

Vstovsky, G. V.: Revelation of spatial and temporal hierarchical structures in complex systems, in: Fluctuations and noise in complex systems of living and dead neture, edited by: Yul'metiev, R. M., Mokshin, A. V., Demin, S. A., Salakhov, M. Kh., Tatarstan Republic Ministry of Education and Science. Editorial and Publishing Center "Shkola”, Kazan' City, 441-454, 2008.
Vstovsky, G. V.: Factual Revelation of Correlation Lengths Hierarchy in Micro- and Nanostructures by Scanning Probe Microscopy Data, Mater. Sci. (Kaunas), 12(3), 262-270, 2006.

Zoller, G. and Hainzl, S.: A systematic spatiotemporal test of the critical point hypotesis for large earthquakes, Geophys. Res. Lett., 29, 5391-5394, 2002. 